\title{
A low-carbon oriented probabilistic approach for transmission expansion planning
}

\author{
Jing QIU, Zhaoyang DONG ( $₫)$, Junhua ZHAO, \\ Ke MENG, Fengji LUO, Kit Po WONG, \\ Chao LU
}

\begin{abstract}
Following the deregulation of the power industry, transmission expansion planning (TEP) has become more complicated due to the presence of uncertainties and conflicting objectives in a market environment. Also, the growing concern on global warming highlights the importance of considering carbon pricing policies during TEP. In this paper, a probabilistic TEP approach is proposed with the integration of a chance constrained load curtailment index. The formulated dynamic programming problem is solved by a hybrid solution algorithm in an iterative process. The performance of our approach is demonstrated by case studies on a modified IEEE 14-bus system. Simulation results prove that our approach can provide network planners with comprehensive information regarding effects of uncertainties on TEP schemes, allowing them to adjust planning strategies based on their risk aversion levels or financial constraints.
\end{abstract}

Keywords Power system planning, Emission reduction, Dynamic programming, Risk management

CrossCheck date: 5 January 2015

Received: 2 December 2014 / Accepted: 26 December 2014/ Published online: 3 February 2015

(C) The Author(s) 2015. This article is published with open access at Springerlink.com

J. QIU, J. ZHAO, K. MENG, F. LUO, Centre for Intelligent

Electricity Networks (CIEN), University of Newcastle,

Callaghan, NSW 2308, Australia

Z. DONG, School of Electrical and Information Engineering,

University of Sydney, Sydney, NSW 2006, Australia

$(\triangle)$ e-mail: zydong@ieee.org

K. P. WONG, School of Electrical, Electronics and Computer

Engineering, University of Western Australia, Perth, WA 6009,

Australia

C. LU, Department of Electrical Engineering,

Tsinghua University, Beijing, China

\section{Introduction}

\subsection{Transmission expansion planning}

Transmission expansion planning (TEP) refers to comprehensive studies on determining the time, location, and type of adding new power transmission lines as well as the associated electrical components, in order to ensure the economic, secure, and reliable operations of a power system [1]. There are a variety of factors contributing to the necessity of TEP, including load growth, components' entry or decommissioning, technological improvement, policy incentive, etc. [2]

Following the advent of electricity markets in many countries, power industry has been transformed from a vertically integrated and regulated utility to an unbundled and liberalized structure. This deregulation and restructuring has resulted in fundamental changes to the power system TEP practices [3, 4]. For instance, the emergence of various self-interested market participants such as brokers and independent power producers has made planning difficult due to conflicting objectives and uncertainties in a market environment $[5,6]$.

Moreover, the power sector is one of the biggest emission sources and should take a key responsibility in promoting the coordinated development among economy, energy and environment by mitigating carbon emission [7, 8]. This concern on carbon emission mitigation is driving the need to explore power system planning practices under the mode of low-carbon economy, e.g. network planning to facilitate the integration of renewable energy and/or to encourage clean power outputs. Therefore, power system planning becomes more complicated under the impacts of different emission reduction policies. 


\subsection{Carbon pricing policies}

Some key carbon pricing policies in the world are summarized as follows. In 2003 in the U.S., carbon emission allowances could be traded among American corporations under a voluntary scheme on Chicago Climate Exchange. In October 2003, the EU parliament approved a new emission trading scheme in order to meet its commitment made in the Kyoto Protocol. Following this, an EU emission trading scheme (ETS), which was the largest multinational, greenhouse gas emission trading scheme in the world, was enforced in February 2005. Under the EU ETS, a specific allowance for emission was allocated to each EU member and any excessive allowance could be sold to whom was in need of allowances. Some allowances were permitted to be transferred between countries through joint implementation (JI) or clean development mechanisms (CDM), but these transfers should be validated by the United Nations Framework Convention on Climate Change (UNFCCC). This type of EU ETS is also called cap-and-trade. In April 2007, an emission price of \$15 NZ per carbon equivalent was implemented. In September 2008, the New Zealand ETS was legislated, and it adopted all free allocation without caps. In July 2010, India introduced a nationwide carbon tax of about \$1.07 US on coal, as coal was a major fuel resource for power generation in India. In November 2011, China had a pilot test of carbon trading in seven provinces and a national trading is expected to start in 2016. In July 2012, the Australian government introduced a carbon price of \$23 AU per ton of emitted carbon equivalent, but this carbon price has been phased out.

\subsection{Review of TEP}

Conventional TEP models are formulated as minimizing investment costs of adding new lines. The widely used deterministic and static TEP model in a regulated power utility is defined as $[6,9,10]$ :

Minimize

$\sum_{i, j \in N} C_{i j} \eta_{i j}+\lambda \sum_{i \in N} r_{i}$

subject to

$$
\begin{aligned}
& \boldsymbol{S}^{\mathrm{T}} \boldsymbol{F}+\boldsymbol{g}+\boldsymbol{r}=\boldsymbol{L} \\
& F_{i j}-B_{i j}\left(\eta_{i j}^{0}+\eta_{i j}\right)\left(\theta_{i}-\theta_{j}\right)=0 \\
& \left|F_{i j}\right| \leq\left(\eta_{i j}^{0}+\eta_{i j}\right) \bar{F}_{i j} \\
& 0 \leq \boldsymbol{g} \leq \overline{\boldsymbol{g}} \\
& 0 \leq \boldsymbol{r} \leq \boldsymbol{L}
\end{aligned}
$$

$0 \leq \eta_{i j} \leq \bar{\eta}_{i j}$

$\eta_{i j}$ is integer, and $i, j \in N$

where $C_{i j}$ is the investment cost of a line added to corridor $i-j ; \eta_{i j}^{0}$ and $\eta_{i j}$ are the initial number of lines between $i-j$ and the number of new added lines; $r_{i}$ is load curtailment at bus $i$ due to insufficient transmission capacity, associated with a penalty factor $\lambda ; S$ is the branch incidence matrix; vectors $\boldsymbol{F}, \boldsymbol{g}, \boldsymbol{r}, \boldsymbol{L}$ denote active power flow, active power output of generators, load curtailment and predicted load, with elements of $F_{i j}, g_{i}, r_{i}, L_{i}$ respectively; $B_{i j}$ denotes the susceptance of a line between $i-j ; \theta_{i}$ is the phase angle at bus $i ; \overline{(\bullet)}$ denotes the upper bounds; $N$ is the total bus number.

To obtain the value of $r_{i}$, usually an optimal power flow (OPF) model is employed to reschedule power generations and alleviate violations of network constraints. The objective of this OPF model is to minimize the total load curtailment [11]. Note this penalty factor for $r_{i}$ helps the optimization process in (1) to find an economical planning solution without a loss of load.

However, the above model is more suitable for a vertically regulated power system, as they do not take into account the market interactions among various stakeholders, e.g. congestion costs caused by different marginal generation costs [12]. By contrast, the main objective of TEP in a new deregulated environment is to provide all stakeholders with nondiscriminatory access to cheap, secure and clean energy resources, subject to reliability and other criteria [13].

\subsection{Contributions of this paper}

The key contributions of the paper are:

1) A chanced constrained load curtailment index is proposed;

2) A risk based probabilistic TEP model is proposed with the consideration of planning uncertainties;

3) A novel hybrid solution algorithm in an iterative process is proposed to solve the formulated multistage programming problem.

\section{Formulated probabilistic model}

\subsection{Define probabilistic load curtailment}

In order to consider uncertainties in TEP, we propose a probabilistic formulation to reflect the level of load curtailment bounded by a threshold, $r_{\max }$. This load curtailment threshold is common in industrial practice. For instance, in Australia, expected energy not supplied (EENS) should be less than $0.002 \%$ of total energy 
consumption, which is also the network planning criterion used by the Australian Energy Market Operator (AEMO) [14]. In our probabilistic approach, the loss of load item in (1) is replaced by a percentage of having load curtailment over the threshold, as given in (9) and (10).

Note the meaning of $\lambda_{R}$ is different from $\lambda$. In (1), $\lambda$ is the cost of each unit of load loss, whereas $\lambda_{R}$ is just a penalty factor and without special meaning, whose unit could be set as $\$$. With this penalty factor, any TEP schemes with or without excessive load curtailment can be included in the optimization process, helping search a bigger solution region and avoiding premature convergence to a local optima.

$\sum_{i, j \in N} C_{i j} \eta_{i j}+\lambda_{R} R$

$R= \begin{cases}0 & \sum_{i \in N_{L}} r_{i} \leq r_{\max } \\ \frac{\sum_{i \in N_{L}} r_{i}-r_{\max }}{r_{\max }} & \sum_{i \in N_{L}} r_{i}>r_{\max }\end{cases}$

When TEP takes into account a variety of uncertainties such as load and wind power output, a commonly used approach is using Monte Carlo (MC) simulations to sample uncertainty scenarios. After simulation stops, EENS is obtained as the average or mean of unsupplied power in the simulation after solving the optimal power flow problems. Planning schemes selected by this method are optimal in the statistical sense, leading to low-probability scenarios being discounted [6]. Moreover, in practice, TEP investment costs may be very high to guarantee that there is no excessive load curtailment at all times.

Alternatively, in this paper, we propose to find a planning scheme which can attain an acceptable probability of nonexcessive load curtailment within system operational constraints. The second term in (9) is transferred into a probability version and this probability is required to be higher than a specified criterion $\alpha$, as shown in (11). This probabilistic approach is rational particularly when network planners are subject to financial constraints or have different risk aversion levels. After that, a chanced constrained index $\varepsilon$ is defined as shown in (12). This index becomes a new objective that should be included into the optimization process.

$$
\begin{array}{ll}
\operatorname{Pr}\left\{\sum_{i \in N_{L}} r_{i} \leq r_{\max }\right\} \geq \alpha & \operatorname{Pr}\left\{\sum_{i \in N_{L}} r_{i} \leq r_{\max }\right\} \geq \alpha \\
\varepsilon= \begin{cases}0 & \operatorname{Pr}\left\{\sum_{i \in N_{L}} r_{i} \leq r_{\max }\right\}<\alpha \\
\alpha-\operatorname{Pr}\left\{\sum_{i \in N_{L}} r_{i} \leq r_{\max }\right\} & \end{cases}
\end{array}
$$

\subsection{Carbon emission modelling}

As summarized in Section 1.2, in general there are two types of carbon pricing policies: mode 1 is carbon tax; mode 2 is carbon trading [15], [16, 17]. In mode 1, only when the annual carbon emission exceeds free emission allowances, power generators will incur a cost for the excessive emission based on the carbon price. In mode 2, power generators can either sell their emission allowance surpluses or by the shortage in allowance from the emission trading market. Therefore, there will be some operating benefits for clean power generators that emit less than their emission allowance. On the other hand, power generators whose annual emission is greater than allowance will incur higher operating costs from buying extra emission allowance. Detailed mathematical formulations of the two emission pricing modes are given in (13) and (14). Note that in (14) $\Phi_{i, t}$ can be either positive or negative value, representing cost or benefit from emission trading.

$\Phi_{i, t}= \begin{cases}0 & \sum_{k=1}^{N_{D}} \delta_{i} D_{k, t} g_{i, k, t} \leq A_{i, t} \\ \sum_{k=1}^{N_{D}} \delta_{i} D_{k, t} g_{i, k, t}-A_{i, t} & \sum_{k=1}^{N_{D}} \delta_{i} D_{k, t} g_{i, k, t}>A_{i, t}\end{cases}$

$\Phi_{i, t}=\sum_{k-1}^{N_{D}} \delta_{i} D_{k, t} g_{i, k, t}-A_{i, t}$

where $\Phi_{i, t}$ and $A_{i, t}$ are the net annual emission and the annual free allowance for generator $i$ in year $t ; \delta_{i}$ is the emission coefficient of the generators; $g_{i, k, t}$ is the power output of generator $i$ at load block $k$ in year $t ; D_{k, t}$ is the duration of load block $k$ in year $t ; N_{D}$ is the total number of load blocks.

\subsection{Uncertainties modelling}

In this paper, uncertainties taken into account are wind power outputs, component working state of the power system, load growth and carbon price. Four types of probability density functions (PDFs) are used to model those uncertainties. Parameters of these PDFs can be obtained based on historical data. MC simulations are deployed to randomly generated scenarios composed by values from the four PDFs.

Wind speed $V$ is generally modeled by a Weibull distribution [18, 19]. Wind power outputs can be derived by wind speed and a power curve of wind power generators. A piecewise function as given in (15) is widely used to model a power curve [20]. 
$P_{W}= \begin{cases}0 & 0 \leq V<V_{\text {In }} \\ P_{W}^{\text {rate }} \frac{V-V_{\text {In }}}{V_{\text {rate }}-V_{\text {In }}} & V_{\text {In }} \leq V<V_{\text {rate }} \\ P_{W}^{\text {rate }} & V_{\text {rate }} \leq V \leq V_{\text {Out }} \\ 0 & V_{\text {Out }}<V\end{cases}$

where $P_{W}$ is the power output of wind power generators, whose rated power output is $P_{W}^{\text {rate }} ; V_{I n}, V_{\text {rate }}, V_{\text {Out }}$ are cutin, rated and cut-out wind speeds, respectively.

The PDF of wind power output i.e. $P_{\text {pdf }}$ is given in (16). $\operatorname{Pr}_{W}^{z e r o}, \operatorname{Pr}_{W}^{r a t e}$ denote probabilities of zero and rated wind power output. $\delta(\bullet)$ is the Dirac delta function used to represent the discrete wind output PDF. $y(\bullet)$ can be expressed by either a fitted polynomial function or discrete samples [9].

$P_{\mathrm{pdf}}\left(P_{W}\right)= \begin{cases}\operatorname{Pr}_{W}^{\text {zero }} \delta\left(P_{W}\right) & P_{W}=0 \\ y\left(P_{W}\right) & 0<P_{W}<P_{W}^{\text {rate }} \\ \operatorname{Pr}_{W}^{\text {rate }} \delta\left(P_{W}-P_{W}^{r a t e}\right) & P_{W}=P_{W}^{\text {rate }} \\ 0 & \text { otherwise }\end{cases}$

The availability of power system components is modelled by a Binomial PDF [21]. The probability $\operatorname{Pr}^{\text {state }}$ of a availability state is given in (17) and (18). $\Omega_{\text {down }}^{\text {state }}, \Omega_{\text {up }}^{\text {state }}$ denote sets of components in unavailable and available states. $r_{F O R}$ is the forced outage rate of components.

$\operatorname{Pr}^{\text {state }}=\prod_{i \in \Omega_{\text {down }}^{\text {state }}}\left(1-\operatorname{Pr}_{i}\right) \prod_{j \in \Omega_{\text {up }}^{\text {state }}}\left(\operatorname{Pr}_{j}\right)$

$\operatorname{Pr}_{j}=1-r_{F O R}$

Uncertainties of load $L$ are modelled by a Normal PDF with mean $\mu$ and standard deviation $\sigma$, as given in (19) [18].

$P_{\mathrm{pdf}}(l ; \mu, \sigma)=\frac{1}{\sqrt{2 \pi \sigma^{2}}} \exp \left[-\frac{(L-\mu)^{2}}{2 \sigma^{2}}\right]$

Uncertainties of carbon price $\phi$ are modelled by a Weibull PDF with scale parameter $\tau$ and shape parameter $\rho$, as given in (20) [15].

$P_{\mathrm{pdf}}(\varphi ; \tau, \rho)=\frac{\rho}{\tau}\left(\frac{\varphi}{\tau}\right)^{\rho-1} \exp \left[-\left(\frac{\varphi}{\tau}\right)^{\rho}\right]$

\subsection{Proposed probabilistic model}

A multi-stage probabilistic TEP model is formulated as minimizing the total cost with the chance constrained index $\varepsilon_{t}$ proposed in Section 2.1, as follows.

Minimize

$$
\begin{array}{r}
\sum_{t=1}^{T} \sum_{i, j \in N} \frac{C_{i j}\left(\eta_{i j, t}-\eta_{i j, t-1}\right)}{(1+\gamma)^{t-1}}+\sum_{t=1}^{T} \frac{\lambda_{\varepsilon} \varepsilon_{t}}{(1+\gamma)^{t-1}} \\
+\sum_{t=1}^{T} \sum_{k=1}^{N_{D}} \sum_{i=1}^{N_{G}} \frac{D_{k, t} C_{O i} g_{i, k, t}}{(1+\gamma)^{t-1}}+\sum_{t=1}^{T} \sum_{i=1}^{N_{G}} \frac{\varphi \Phi_{i, t}}{(1+\gamma)^{t-1}}
\end{array}
$$

subject to

$$
\begin{aligned}
& \sum_{i, j \in N} F_{i j, k, t}+\sum_{i \in N_{G}} g_{i, k, t}+\sum_{i \in N_{L}} r_{i, k, t}-\sum_{i \in N_{L}} L_{i, k, t}=0 \\
& F_{i j, k, t}-B_{i j}\left(\eta_{i j, t}^{0}+\eta_{i j, t}\right)\left(\theta_{i, k, t}-\theta_{j, k, t}\right)=0 \\
& \left|F_{i j, k, t}\right| \leq\left(\eta_{i j, t-1}+\eta_{i j, t}\right) \bar{F}_{i j, k, t}
\end{aligned}
$$

$0 \leq g_{i, k, t} \leq \bar{g}_{i}$

$0 \leq r_{i, k, t} \leq L_{i, k, t}$

$0 \leq \eta_{i j, t} \leq \bar{\eta}_{i j}$

$\eta_{i j, t}$ is integer, and $i, j \in N$

Equation (21) is a model represented by net present value (NPV) with a discount rate $\gamma . T$ is the total planning horizon. $i, j$ are superscripts for buses. $k, t$ are superscripts for load block and planning year respectively. The first term is the investment cost, and the second term is the chance constrained index at year $t$ scaled by a penalty factor $\lambda_{\varepsilon}$. The third term is the operating cost of power generators, whose incremental cost of output is denoted by $C_{O i}$. The total number of generators in the system is $N_{G}$. The fourth term is the cost of carbon emission, derived from carbon price and the net annual emission of power generators.

For completeness, we use a piecewise function to derive the quadratic losses in the DC power model, as given in (29)-(37). Note losses are not incorporated into the objective function. Instead, they are considered as the additional active power in (22) required from power generation, in order to satisfy the nodal balance due to the presence of losses.

The detailed linearization process is given as follow.

$$
\begin{aligned}
& P_{i j, k, t}^{\text {loss }} \approx 2 G_{\text {condij }}\left(1-\cos \theta_{i-j, k, t}\right) \\
& \theta_{i-j, k, t}^{2} \approx \sum_{h=1}^{H} \varpi(h) \Delta \theta_{i-j, k, t}(h) \\
& \varpi(h)=(2 h-1) \frac{\theta_{i-j, t}^{\max }}{H} \\
& \theta_{i-j, k, t}=\theta_{i-j, k, t}^{+}-\theta_{i-j, k, t}^{-}
\end{aligned}
$$

$\left|\theta_{i-j, k, t}\right|=\sum_{h=1}^{H} \Delta \theta_{i-j, k, t}(h)=\theta_{i-j, k, t}^{+}+\theta_{i-j, k, t}^{-}$

$0 \leq \theta_{i-j, k, t}^{+} \leq \chi \theta_{i-j, t}^{\max }$

$0 \leq \theta_{i-j, k, t}^{-} \leq(1-\chi) \theta_{i-j, t}^{\max }$

$0 \leq \Delta \theta_{i-j, k, t}(h) \leq \frac{\theta_{i-j, t}^{\max }}{H}$

$0 \leq \Delta \theta_{i-j, k, t}(h) \leq \Delta \theta_{i-j, k, t}(h-1)$ 


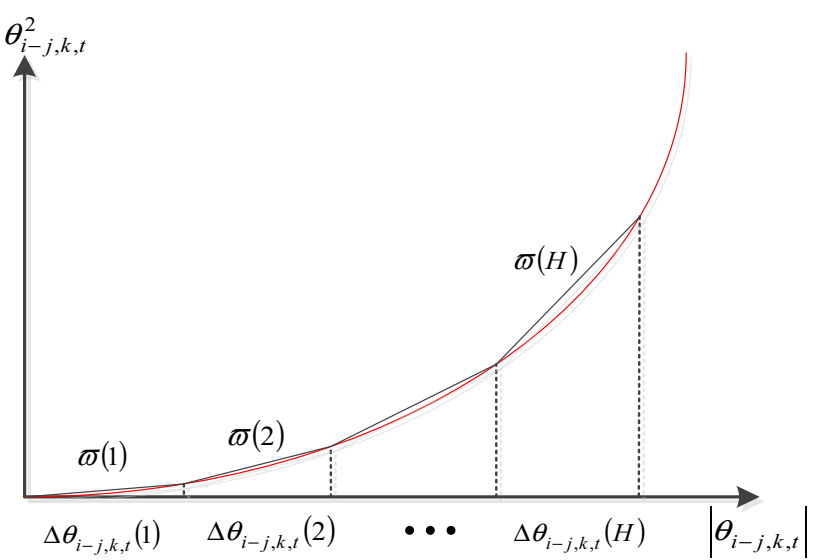

Fig. 1 Piecewise linear approximation of phase angle difference between $i-j$

Where $P_{i j, k, t}^{l o s s}, \theta_{i-j, k, t}$ are the active power losses and phase angle difference between $i-j$ at load block $k$ in year $t$, repectively; $G_{\text {cond } i j}$ is the line conductance; the quadratic term $\theta_{i-j, k, t}^{2}$ is linearized by a piecewise function, as shown in Fig. $1 ; H$ is the total number of intersection, with a $h$-th intersection of $\Delta \theta_{i-j, k, t}(h)$, and the slope is $\varpi(h)$; two nonnegative slack variables $\theta_{i-j, k, t}^{+}, \theta_{i-j, k, t}^{-}$are introduced to replace $\theta_{i-j, k, t}$, and they are also bounded by a binary variable $\chi$ to make this replacement effective, as shown in (32)-(35). Equations (36)-(37) can ensure the linear intersection on the left side is always filled up first.

\section{Solution algorithms}

Solution algorithms for TEP problems mainly fall into either mathematical programming classes or heuristic search classes. Mathematical programming methods have strict requirements on the model itself (e.g. the problem or the continuous relaxation of the problem should be convex) and can provide more clues on the quality of the final solution [22]. However, mathematical programming methods tend to be trapped by local optima in some cases. On the contrary, heuristic methods are suitable for stochastic global search, free from problem formulation difficulties and can escape from premature local optimal. The drawbacks of heuristic methods are: the quality of the solution cannot be guaranteed; and prohibitive computation efforts are required [22, 23, 24].

In this paper, the proposed TEP model is a dynamic optimization problem with a chance constrained reliability evaluation. To enhance the solution performance of the proposed model, a hybrid method based on decomposition is proposed as follows.

The overall stochastic programming problem can be divided into two subproblems: 1) the investment

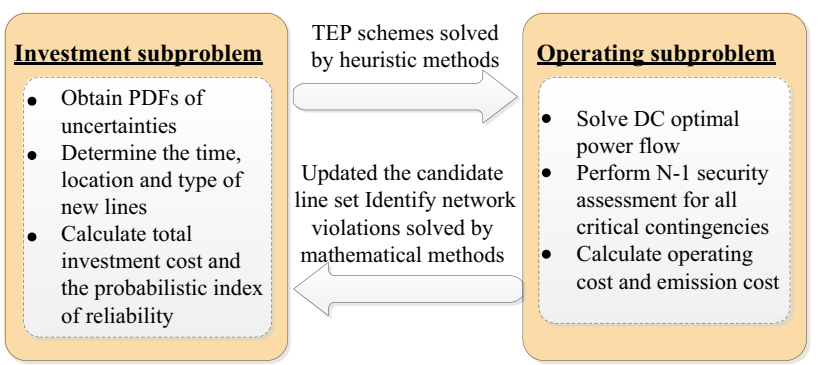

Fig. 2 An iterative solution algorithm for the decomposed two subproblems

subproblem in the first and second terms of the defined objective; 2) the operating subproblem in the third and fourth terms of the defined objective. Note that the investment subproblem is targeted at a specific planning year, whereas the operating decisions are subject to the decisions from the investment subproblem and should be evaluated over multiple years thereafter [25].

Firstly, an evolutionary algorithm is deployed to help the optimization process in subproblem 1 to find a bigger set of expansion candidates. In this paper, differential evolution (DE) is used, as it is shown faster, simpler and more robust [6]. Secondly, the subproblem 2 can be soundly solved by a state-of-the-art nonlinear programming technique, such as the interior point (IP) method. IP is widely used in solving power system operation and dispatch problems, because the optimality of the solution found by IP is guaranteed [26]. Moreover, IP has higher efficiency in searching local optima [26], which means that IP can quickly locate local optima for the subproblem 2, adding them to new generation in the evolution process. As shown in Fig. 2, the two subproblems are solved in an iterative way to progressively converge to the final solution. The termination criterion can be defined by two ways: the maximum iteration number is reached; or the objective value does not improve for a few successive generations.

The main procedure of the proposed hybrid method is given as follows:

1) Determine the PDFs of wind speed, carbon price, load, and FOR based on historical data. Note that the PDF of wind power outputs is translated from the wind power curve in (15).

2) MC simulations are deployed to generate values of wind power outputs, load, component availability, and carbon price from the PDFs in step 1).

3) Initialize the population of the EA corresponding to the number of new added lines. Note that the quality of initial population has great impacts on the final solution. Therefore, in this process, uncertain features are neglected and some deterministic values are assigned. To be specific, carbon price is set to its 
mean value, wind power output is set to the installed capacity scaled by its capacity factor, all components are set as available, and load is set as $(\mu+3 \sigma)$.

4) Using the initial values generated by step 3 ), denoted by $\boldsymbol{\eta}^{G}=\left\{\eta_{i j, 0}, \forall i, j \in N\right\}$, apply the IP to generate local optima, i.e. subproblem 2 is solved with this generation. In this process, IP can solve the DC optimal power flow (OPF) for each individual in the population. Note if network violations are identified, a linear programming is required to minimize the total load curtailment, i.e. re-dispatching of generation is not based on bids or marginal costs. Then calculate the operating and carbon emission costs and add the two local optima to $\boldsymbol{\eta}^{G+1}$. Also, based on identify network violations, find the probability of $\operatorname{Pr}\left\{\sum_{i \in N_{L}} r_{i} \leq r_{\max }\right\}$, and calculate the probabilistic index of reliability.

5) Start mutation and recombination process. Integers representing adding or reducing lines will be generated and recombined.

6) For each TEP scheme, the objective function in (21) is assigned as the fitness value. A newly generated child individual is compared with the parent, and replaces it if the fitness value of the child is smaller.

7) Terminate the algorithm if the stopping criterion is satisfied, otherwise go back to step 2).

\section{Case studies}

In this section, a series of numerical experiments are undertaken to demonstrate the performance of the proposed model on the modified IEEE 14-bus system. The 14-bus system initially has five power generators, and 20 transmission corridors.

In our paper, the five power generators in the base case are assumed to be fossil-fuel-fired, and the carbon emission coefficients for them are set as 1.2, 1, 0.8, 0.8 and $0.6 \mathrm{tCO}_{2} /$ MWh respectively. Other power generation types such as hydro power or nuclear power could be included as future works. Moreover, for simplicity, power generators are assumed to receive $80 \%$ of their emission allowances for free based on their emissions in the base year, and free allowances will decrease linearly each year to $30 \%$ in the last planning horizon. Note that allocating emission allowances is a complex issue involving political motivation. The assumption made in this paper regarding emission allocation is in accordance with the EU ETS practice, which can be found in [27].

The total generation capacity of the base system is 720 MW, while the total load is 440 MW. Durations for load

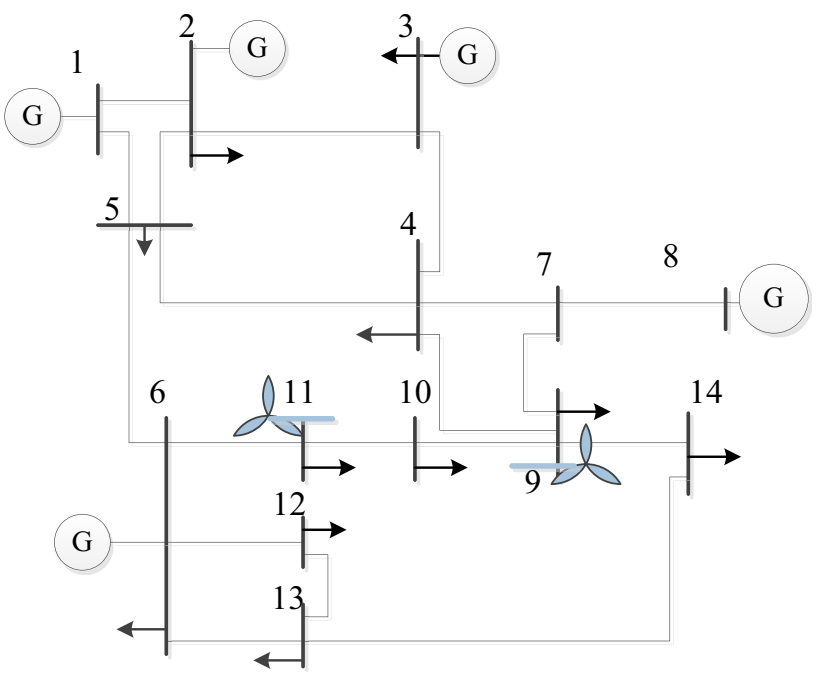

Fig. 3 Modified IEEE 14-bus system with two wind power generators

blocks are 20\%, 50\%, 29\% and 1\%. As illustrated in Fig. 3, two wind farms with capacities of $50 \mathrm{MW}$ are assumed to be installed at buses 9 and 11. The two wind farms are assumed to be totally independent, and therefore the wind speeds for modelling their outputs are randomly generated from two different Weibull distribution functions. Parameters for modelling the wind power curve are $V_{I n}=4$, $V_{\text {rate }}=10, V_{\text {Out }}=22 \mathrm{~m} / \mathrm{s}$. To model the possibility of $\mathrm{N}-1$ contingencies, transmission lines outages are modelled by a $r_{F O R}$ of $1 \%$. The mean value of carbon prices is set as $E[\varphi]=\$ 23 / \mathrm{tCO}_{2}$. The planning horizon is set as five years, and the annual load growth rate is $5 \%$, with uncertainties of $\sigma / \mu=2 \%$, i.e. $\mu=0.05, \sigma=0.001$.

The capacity of each candidate line is $100 \mathrm{MW}$, and up to four lines are allowed for each corridor. The investment cost is assumed to be $50 \mathrm{M} \$ / 100 \mathrm{~km}$. We set the upper bound of load curtailment threshold as a percentage of the total annual energy demand, i.e. $r_{\max }=0.1 \%$. To make $\varepsilon_{t}$ have a significant effect in the objective function, the penalty factor $\lambda_{\varepsilon}$ is set big enough to ensure that planning schemes with considerable load curtailments will be eliminated in the optimization process during the heuristic search. The settings of $\alpha$ and $\lambda_{\varepsilon}$ are 95\% and $\$ 1 \times 10^{9}$.

In order to make a comparison and demonstrate the performance of the proposed probabilistic approach, three deterministic TEP studies with different carbon pricing policies are undertaken, i.e. deterministic TEP with no emission price, deterministic TEP in carbon mode 1 and mode 2 respectively with a carbon price of $\$ 23 / \mathrm{tCO}_{2}$. As illustrated in Table 1, investment costs for three deterministic models are all $378.25 \mathrm{M} \$$, whereas the investment cost for Case 4 is the highest due to the volatility of emission market. For operating cost, Case 1 is the lowest as 
Table 1 Comparisons of deterministic and probabilistic TEP with different carbon pricing policies

\begin{tabular}{llllr}
\hline Case No & Total cost $(\mathrm{M} \$)$ & Invest. cost $(\mathrm{M} \$)$ & Operating cost $(\mathrm{M} \$)$ & Emission cost $(\mathrm{M} \$)$ \\
\hline Case 1: deterministic TEP with no emission price & 890.36 & 378.25 & 512.11 & 0 \\
Case 2: deterministic TEP in mode 1 with $\$ 23 / \mathrm{tCO}_{2}$ & 1119.22 & 378.25 & 615.62 & 125.35 \\
Case 3: deterministic TEP in mode 2 with $\$ 23 / \mathrm{tCO}_{2}$ & 1061.48 & 378.25 & 572.98 & 110.25 \\
Case 4: probabilistic TEP in mode 2 & 1107.77 & 415.64 & 586.77 & 105.36 \\
\hline
\end{tabular}

a result of no carbon pricing policies. Moreover, compared to Case 2, the operating cost in Case 3 is lower. This is because some power generators can earn benefits in the emission trading market if they have lower emission coefficient. Although the carbon price uncertainty increases the investment cost in Case 4, the total cost in Case 4 is lower compared to that in Case $2(1,107.77$ vs $1,119.22$ $\mathrm{M} \$$ ), reflecting the operating benefits of carbon mode 2. It should be noted that, if stochastic features are removed, i.e. without uncertainties of wind power, load and carbon price, for zero load curtailment and selected line outages, the results of the probabilistic and deterministic TEP are identical, which validates the applicability of our approach. Furthermore, comparisons in Table 1 reveal that the planning schemes identified by deterministic TEP are greatly affected by carbon price uncertainties, leading to a high probability of load curtailment. This may imply that the deterministic TEP is insufficient, particularly when exposed to higher risks of uncertainties.

As mentioned in Section 2.1, the probability $\alpha$ is defined as the lower bound for each planning scheme the possibility of having load curtailment below the required threshold, and $\alpha \in[0,1]$. This probability can be interpreted as a risk measure, which can be chosen by network planners. The higher value of $\alpha$, the more important to choose a plan without excessive load curtailment, leading to a higher investment cost (more conservative). We set $\alpha$ as three different levels to run simulations of Case 4 . The probabilities of load curtailment are given in Fig. 4. As seen, a plan chosen by a higher $\alpha$ is said to be risk averse, whereas a plan chosen by lower $\alpha$ is said to be risk preferring. Note a higher $\alpha$ also increases the probability of no load curtailment (see the intersections on the $Y$-axis in Fig. 4). In this way, our probabilistic approach can provide comprehensive information for network planners regarding the balance between minimizing investment costs and minimizing the risk of load curtailment in the face of uncertainties.

The detailed result of our probabilistic TEP in carbon mode 2 is given in left column in Table 2 . As given in the right two columns in Table 2, carbon price uncertainties (i.e. high standard deviation) make a planning scheme expose to higher risk, requiring higher investment cost for

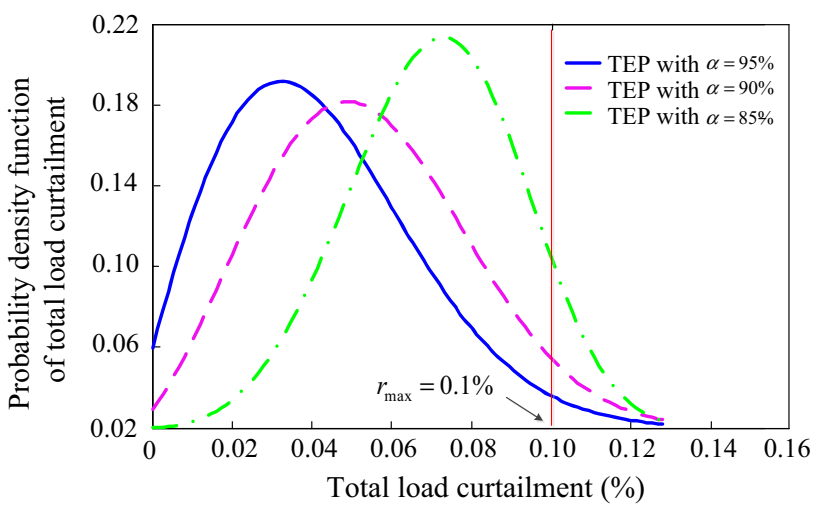

Fig. 4 Probability of load curtailment with different $\alpha$ values

meeting the $\alpha$ criterion (the initial $\alpha$ is set as $95 \%$ ). As seen, if the standard deviation of carbon prices is set above $\$ 3 /$ $\mathrm{tCO}_{2}$, investment costs have to be increased as $\alpha$ becomes lower than $95 \%$. Also, the same situation will happen when the mean of carbon prices are raised from $\$ 23 / \mathrm{tCO}_{2}$ to $\$ 35 /$ $\mathrm{tCO}_{2}$, which implies that more use of intermittent renewable energy (e.g. wind power), will increase TEP uncertainties, thus requiring higher investment costs. These findings are also supported by simulation results in Fig. 5 . According to Fig. 5, the total costs without carbon price or with fixed carbon price are always lower than total costs with carbon price uncertainties in both modes 1 and 2, regardless of the value of $\alpha$.

To examine the effects of different upper bounds of the maximum load curtailment parameter $r_{\max }$, we run simulations to obtain the total cost with different values of $\alpha$. As shown in Fig. 6, the higher $r_{\max }$, the lower total cost. This is more obvious with a conservative planning scheme, i.e. with a higher $\alpha$. In addition, results of the total cost against total installed wind power capacity with different $\alpha$ values are given in Fig. 7. As seen, for a specific $\alpha$, it is possible to obtain the optimal wind power capacity, i.e. the lowest total cost when integrating different wind power capacities into the system. For instance, when $\alpha$ is chosen as $95 \%$, in order to obtain the lowest total cost, the optimal wind power capacity to be integrated into the power system should be about $60 \mathrm{MW}$. This finding is of significance for renewable energy integration analysis, as the rapid growth 
Table 2 Final result against different carbon price characteristics

\begin{tabular}{lll}
\hline Lines added & $\begin{array}{l}\text { Different carbon price } \\
\text { characteristics }(\$ / \mathrm{tCO})\end{array}$ & $\alpha$ \\
\hline$\eta_{1-2, t=2}=1 ; \eta_{4-10, t=2}=1 ; \eta_{7-9, t=2}=1 ; \eta_{6-11, t=3}=2 ; \eta_{10-11, t=3}=1 ;$ & Mean is 23; std. is 3 & 0.9575 \\
$\eta_{12-13, t=3}=1 ; \eta_{14-9, t=3}=1 ; \eta_{2-3, t=4}=2 ; \eta_{3-4, t=4}=3 ; \eta_{1-2, t=5}=1 ;$ & Mean is 23; std. is 5 & Mean is 35; td. is 3 \\
$\eta_{1-5, t=5}=1 ; \eta_{5-6, t=5}=4 ; \eta_{6-11, t=5}=2 ; \eta_{12-13, t=5}=1$ & Mean is 35; std. is 5 & 0.9028 \\
\end{tabular}

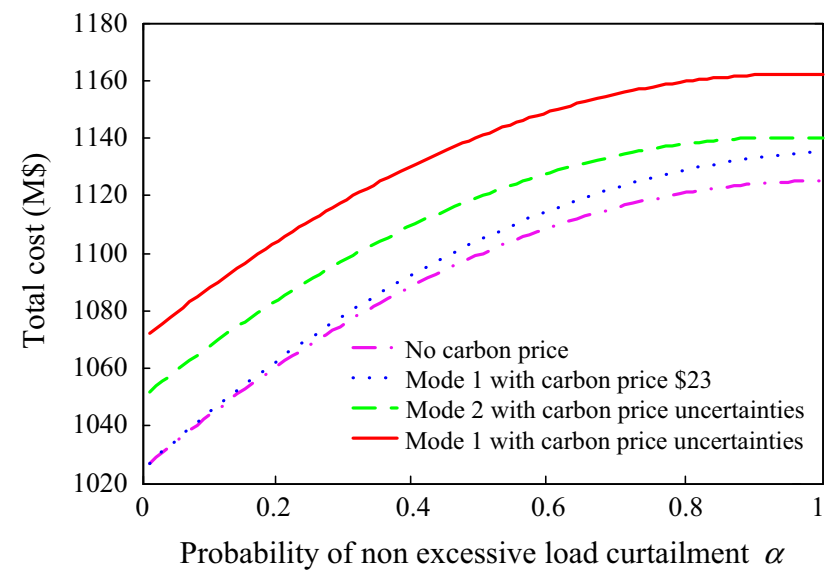

Fig. 5 Total cost against different values of $\alpha$ with different carbon uncertainties

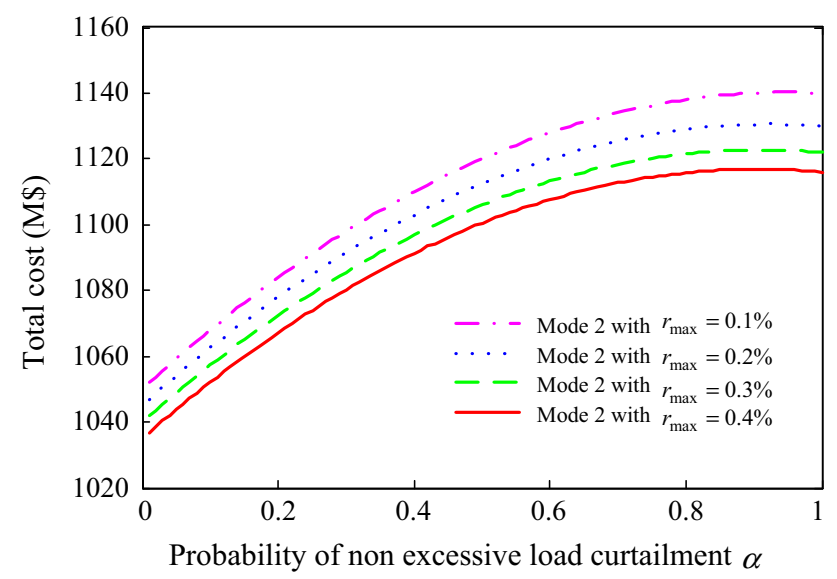

Fig. 6 Total cost against different values of $\alpha$ with different total load curtailment thresholds

of wind power will have significant impact on power system operations and planning [28]. Moreover, our approach can reveal the relationship between the overall system cost and the risk of load curtailment with the presence of wind power uncertainties. Therefore, our probabilistic TEP model serves a good indicative role in achieving low carbon economy, in terms of identifying a robust and costeffective planning scheme.

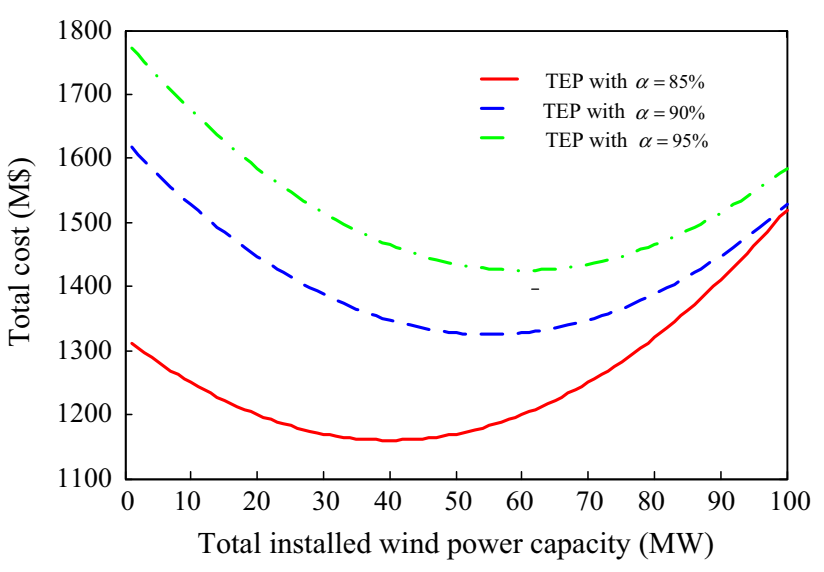

Fig. 7 Total cost against different total installed wind power capacity with different values of $\alpha$

\section{Conclusion}

This paper has proposed a probabilistic TEP model with a chance constrained load curtailment index. Planning uncertainties such as wind power output, component availability, load, and carbon price are incorporated by a Monte Carlo simulation based approach. Our multi-stage planning objective is formulated as minimizing the total cost, including investment cost, operating cost, emission cost and a risk factor of load curtailment. For completeness, a piecewise approximation function is used to linearize the quadratic power losses. Meanwhile, a novel iterative solution algorithm combing heuristic search and mathematical programming is proposed to solve the formulated a dynamic optimization problem. The performance of the proposed approach is demonstrated by a modified IEEE 14-bus system. Simulation results have proved that our approach can give network planners an opportunity to trade-off between the overall cost and the probability of load curtailment in the presence of uncertainties. Our approach can also provide network planners with comprehensive information regarding effects of uncertainties on TEP schemes, allowing them to adjust planning strategies based on their risk aversion levels or financial constraints. Moreover, our approach can be used for renewable energy integration analysis in terms of long-term network planning. Therefore, our novel TEP approach is a risk-based, flexible decision 
tool, which is important for achieving low carbon economy through planning practices.

Open Access This article is distributed under the terms of the Creative Commons Attribution License which permits any use, distribution, and reproduction in any medium, provided the original author(s) and the source are credited.

\section{References}

[1] Buygi MO, Balzer G, Shanechi HM, Shahidehpour M (2004) Market-based transmission expansion planning. IEEE Trans Power Syst 19(4):2060-2067

[2] Garces LP, Conejo LJ, Garcia-Bertrand R, Romero R (2009) A bilevel approach to transmission expansion planning within a market environment. IEEE Trans Power Syst 24(3):1513-1522

[3] Escobar AH, Gallego RA, Romero R (2004) Multistage and coordinated planning of the expansion of transmission systems. IEEE Trans Power Syst 19(2):735-744

[4] Zhang F, Hu Z, Song Y (2013) Mixed-integer linear model for transmission expansion planning with line losses and energy storage systems. IET Gen Trans \& Dist 7(8):919-928

[5] Heejung P, Baldick R (2013) Transmission planning under uncertainties of wind and load: sequential approximation approach. IEEE Trans Power Syst 28(3):2395-2402

[6] Zhao JH, Dong ZY, Lindsay P, Wong KP (2009) Flexible transmission expansion planning with uncertainties in an electricity Market. IEEE Trans Power Syst 24(1):479-488

[7] Tan ZF, Ngan HW, Yang Wu, Zhang HJ, Song YH, Yu C (2013) Potential and policy issues for sustainable development of wind power in China. J Mod Power Syst Clean Energy 1(3):204-215

[8] Huang J, Xue F, Song XF (2013) Simulation analysis on policy interaction effects between emission trading and renewable energy susidy. J Mod Power Syst Clean Energy 1(2):195-201

[9] Yu H, Chung CY, Wong KP, Zhang JH (2009) A chance constrained transmission network expansion planning method with consideration of load and wind farm uncertainties. IEEE Trans Power Syst 24(3):1568-1576

[10] Xu Z, Dong ZY, Wong KP (2006) A hybrid planning method for transmission networs in a deregulated environment. IEEE Trans Power Syst 21(2):925-932

[11] Guo L, Qiu QW, Liu J, Zhou Y, Jiang LL (2014) Power transmission risk assessment considering component condition. J Mod Power Syst Clean Energy 2(1):50-58

[12] Roh JH, Shahidehpour M, Wu L (2009) Market-based generation and transmission planning with uncertainties. IEEE Trans Power Syst 24(3):1587-1598

[13] Maghouli P, Hosseini SH, Buygi MO, Shahidehpour M (2009) A multi-objective framework for transmission expansion planning in deregulated environments. IEEE Trans Power Syst 24(2): 1051-1061

[14] Australian Energy Market Operator (AEMO). http://www.aemo. com.au/. Accessed 18 Oct 2014

[15] Kazerooni AK, Mutale J (2010) Transmission network planning under security and environmental constraints. IEEE Trans Power Syst 25(2):1169-1178

[16] Chen QX, Kang CQ, Xia Q, Zhong J (2010) Power generation expansion planning model towards low-carbon economy and its application in China. IEEE Trans Power Syst 25(2):1117-1125

[17] Zhou X, James G, Liebman A, Dong ZY, Ziser C (2010) Partial carbon permits allocation of potential emission trading scheme in Australian electricity market. IEEE Trans Power Syst 25(1):543-553
[18] Ghofrani M, Arabali A, Etezadi-Amoli M, Fadali MS (2013) Energy storage application for performance enhancement of wind integration. IEEE Trans Power Syst 28(4):4803-4811

[19] Siano P, Mokryani G (2013) Probabilistic assessment of the impact of wind energy integration into distribution networks. IEEE Trans Power Syst 28(4):4209-4217

[20] Orfanos GA, Georgilakis P, Hatziargyriou ND (2013) Transmission expansion planning of systems with increasing wind power integration. IEEE Trans Power Syst 28(2):1355-1362

[21] Jaeseok C, Mount TD, Thomas RJ, Billinton R (2006) Probabilistic reliability criterion for planning transmission system expansions. IET Gen Trans \& Dist 153(6):719-727

[22] Zhang H, Heydt GT, Vittal V, Quintero J (2013) An improved network model for transmission expansion planning considering reactive power and network losses. IEEE Trans. Power Syst. 28(3):3471-3479

[23] Tekiner H, Coit DW, Felder FA (2010) Multi-period multiobjective electricity generation expansion planning problem with Monte-Carlo simulation. Elec Power Syst Res 80(12): 1394-1405

[24] Zhang H, Vittal V, Heydt GY, Quintero J (2012) A mixedinteger linear programming approach for multi-stage securityconstrained transmission expansion planning. IEEE Trans Power Syst 27(2):1125-1133

[25] Roh JH, Shahidehpour M, Fu Y (2007) Market-based coordination of transmission and generation capacity planning. IEEE Trans Power Syst 22(4):1406-1419

[26] Zhao JH, Wen FS, Dong ZY, Xue YS, Wong KP (2012) Optimal dispatch of electric vehicles and wind power using enhanced particle swarm optimization. IEEE Trans Indus Infor 8(4): 889-898

[27] European Commission on Climate Action. http://ec.europa.eu/ clima/policies/ets/cap/allocation/index_en.htm. Accessed 21 Oct 2014

[28] Xue YS, Cai B, James G, Dong ZY, Wen FS, Xue F (2014) Primary energy congestion of power systems. J Mod Power Syst Clean Energy 2(1):39-49

Jing QIU (S'12-M'14) is now a Research Associate at the Center for Intelligent Electricity Networks (CIEN), The University of Newcastle, Australia. He obtained his B.Eng. degree in control engineering from Shandong University, China, M.Sc. degree in environmental policy and management, majoring in carbon financing in the power sector, from The University of Manchester, U.K., and Ph.D. in electrical engineering from The University of Newcastle, Australia. His areas of interest include electricity market modeling, power system planning, renewable energy, carbon emission reduction and risk management.

Zhaoyang DONG (M'99-SM'06) obtained his Ph.D. degree from the University of Sydney, Australia in 1999. He is now Professor and Head of School of Electrical and Information Engineering, University of Sydney, Australia. He was previously Ausgrid Chair and Director of the Center for Intelligent Electricity Networks (CIEN), The University of Newcastle, Australia, and is now a conjoint professor there. He also held academic and industrial positions with the Hong Kong Polytechnic University and Transend Networks (now TasNetworks), Tasmania, Australia. His research interest includes Smart Grid, power system planning, power system security, load modeling, electricity market, and computational intelligence and its application in power engineering. Prof. Dong is an editor of the IEEE TRANSACTIONS ON SMART GRID, the IEEE TRANSACTIONS ON SUSTAINABLE ENERGY, IEEE POWER ENGINEERING LETTERS, and IET Renewable Power Generation. 
Junhua ZHAO (S'04-M'07) obtained his B.E. and Ph.D. degrees from Xi'an Jiaotong University, China and The University of Queensland, Australia in 2003 and 2007 respectively. He then worked as a postdoctoral research fellow at the University of Queensland, Australia. He is now a Senior Lecturer in Center for Intelligent Electricity Networks, The University of Newcastle, Australia. His research interests include power system analysis and computation, smart grid, energy Internet, data mining and computational intelligence and their applications in power engineering.

Ke MENG (M'10) obtained Ph.D. from the University of Queensland, Australia in 2009. He is currently with the Centre for Intelligent Electricity Networks (CIEN), The University of Newcastle, Australia. His research interest includes pattern recognition, power system stability analysis, wind power, and energy storage.

Fengji LUO (M'13) obtained the B.S. and M.S. degrees in software engineering from Chongqing University, Chongqing, China, in 2006 and 2009, respectively. He received the Ph.D. degree in electrical engineering from the University of Newcastle, Australia, in 2013. Currently, he is the research associate of the Centre for Intelligent Electricity Networks, Australia. His research interests include computational intelligence applications, distributed computing, and power system operation \& planning.
Kit Po WONG (M'87, SM'90, F'02), obtained M.Sc, Ph.D. and D. Eng degrees from the University of Manchester, Institute of Science and Technology, in 1972, 1974 and 2001 respectively. Since 1974, he has been with the School of Electrical, Electronic and Computer Engineering, The University of Western Australia, Perth, Australia, where he currently is a Winthrop Professor. From 2002 to 2011, he was also with Department of Electrical Engineering,The Hong Kong Polytechnic University, as Chair Professor and Head (2002-2007). He is a con-joint professor of The University of Newcastle, Australia. His current research interests include power system analysis, planning and operations, smart grids and renewable energy. Prof. Wong received three Sir John Madsen Medals (1981, 1982, and 1988) from the Institution of Engineers Australia, the 1999 Outstanding Engineer Award from IEEE Power Chapter Western Australia, and the 2000 IEEE Third Millennium Award. He was the General Chairman of IEEE/CSEE PowerCon2000. He is now Editor-in-Chief of IEEE POWER ENGINEERING LETTERS and was Editor-in-Chief of IEE Proc. Generation, Transmission \& Distribution. He is a Fellow of IEEE, IET, HKIE, and IEAust.

Chao LU obtained his B.E., Ph.D. from Tsinghua University, Beijing, China. He is now associate professor at Tsinghua University, China. His research interest includes power system stability, power system transients and power system planning. 